\title{
Faster colonoscope withdrawal time without impaired detection using EndoRings
}

\section{다 $(9)$}

\author{
Authors \\ John C. Thygesen, Prasanna Ponugoti, William W. Tippins, Jonathan R. Garcia, Andrew W. Sullivan, Heather \\ M. Broadley, Douglas K. Rex
}

Institution

Division of Gastroenterology/Hepatology, Indiana

University School of Medicine, Indianapolis, Indiana, United

States

submitted 30.11 .2017

accepted after revision 22.1.2018

Bibliography

DOI https://doi.org/10.1055/a-0614-2052 |

Endoscopy International Open 2018; 06: E957-E960

(c) Georg Thieme Verlag KG Stuttgart · New York

ISSN 2364-3722

Corresponding author

Douglas K. Rex, MD, Indiana University, 550 North

University Boulevard, Suite 4100, Indianapolis, IN 46202

Fax: +1-3179445449

drex@iu.edu

\section{ABSTRACT}

Background and study aims Mucosal exposure devices on the colonoscope tip have improved detection. We evaluated detection and procedure times in colonoscopies performed with EndoRings.

Patients and methods We had 14 endoscopists in a university practice trial EndoRings. We compared detection and procedure times to age- and indication-matched procedures by the same endoscopists.

Results There were 137 procedures with EndoRings. The adenoma detection rate was $44 \%$ with EndoRings vs. $39 \%$ without $(P=0.39)$. Mean adenomas per colonoscopy (standard deviation) was 1.2 (2.3) with EndoRings vs. 0.9 (1.6) without $(P=0.055)$. Mean insertion time with EndoRings was 6.2 (3.2) minutes vs. 6.6 (6.7) minutes without $(P=$ $0.81)$. Mean withdrawal time with EndoRings in all patients with or without polypectomy was 12.2 (5.3) minutes and 16.1 (10.3) minutes without $(P=0.0005)$.

Conclusion EndoRings may allow faster withdrawal during colonoscopy without any reduction in detection. Prospective trials with mucosal exposure devices targeting procedure times as primary endpoints are warranted.

\section{The adenoma}

Adenoma detection rate (ADR) has emerged as the most important quality measure in colonoscopy [1]. Higher ADRs predict lower risk of interval cancer before the next scheduled colonoscopy [2,3]. Improvements in ADR have been associated with individual physicians reducing risk of interval cancer in their patients over time [4].

Although results have been sometimes inconsistent, ADR has been improved by reporting to physicians [5] or the public [6], by split-dose bowel preparations [7], high-definition colonoscopy [8], chromoendoscopy $[9,10]$, and mucosal exposure devices such as cap on the colonoscope tip [11], Endocuff (Arc Medical Design, Leeds, UK) [12], and EndoRings (EndoAid Ltd, Caesarea, Israel) [13]. These mucosal exposure devices improve detection by allowing the colonoscopist to flatten haustral folds during withdrawal, thereby exposing mucosa on the proximal sides of folds.
As part of our endoscopy unit routine quality improvement efforts, we invited endoscopists to sample EndoRings. We sought to familiarize our endoscopists with a fold flattening device, and potentially improve their individual ADRs. Although the focus of EndoRings is to improve ADR, we observed that the ADR remained stable while there was a substantial reduction in total withdrawal time. In this report we describe the results and propose a mechanism for this occurrence.

\section{Patients and methods}

As part of our continuing quality improvement program, we decided to sample the impact of EndoRings on colonoscopy performance during routine procedures. We asked Endoaid to provide us a sample of 100 devices to be tested by our colonoscopists. We also had some devices left over from a recently completed (as yet unpublished) randomized controlled trial performed by one of our endoscopists. All procedures were 
- Table 1 Comparison of demographic features between EndoRings cases and controls.

\begin{tabular}{|l|l|l|}
\hline & EndoRings & Controls \\
\hline Number of subjects & 137 & 127 \\
\hline Mean age (SD) years & $60.7(9.3)$ & $60.4(8.3)$ \\
\hline Female (\%) & 61 & 61 \\
\hline Screening (\%) & 65 & 65 \\
\hline Surveillance (\%) & 35 & 35 \\
\hline SD, standard deviation & & \\
\hline
\end{tabular}

performed in an outpatient endoscopy center. Each colonoscopist was given brief instruction in the purpose and use of the EndoRings device and instructed not to use the device in patients with known colonic strictures or severe diverticular disease.

Eligible patients were aged $\geq 50$ years with screening or polyp surveillance as the colonoscopy indication. A research assistant recorded patient age, gender, procedure indication, insertion time, whether insertion to the cecum was successful, and the total withdrawal time for the procedure.

A study assistant measured insertion and withdrawal time with a stopwatch. Insertion time was from the moment of the colonoscope insertion into the patient until the colonoscope tip was proximal to the ileocecal valve and fully in the cecal caput. Withdrawal time began when cecal cleaning was finished and cecal inspection started until rectal retroflexion was completed. Neither polypectomy time (if any) nor washing and suctioning time (after initial cecal cleaning) were subtracted from withdrawal time.

After completing the study procedures, we matched each procedure with a second examination. This was the most recent procedure available in our endoscopy unit database performed by the same endoscopist on a patient with the same gender, age (within \pm 2 years), and procedure indication (screening or surveillance). Eligible procedures for matching had to have been performed within 2 years of the index procedure.

As the study was considered an exploratory quality study for our unit, and the device was approved by the US Food and Drug Administration, we did not obtain informed consent from patients for use of the device. Recruitment occurred between March 2016 and September 2016. Permission to review the data for publication was granted by the Institutional Review Board at Indiana University on September 11, 2017.

\section{Statistical analysis}

Age was compared between EndoRings patients and controls using the two-sample $t$-test, and gender and indication by the chi-square test. ADR and polyp detection rate (PDR; percentage of subjects with one or more polyps removed) were compared by logistic regression, with age and sex as covariates. Adenomas per colonoscopy (APC) and polyps per colonoscopy (PPC) were compared by negative binomial regression for count data, with age and sex included as covariates. Insertion and withdrawal times were compared using ANCOVA, with age and sex included as covariates.

\section{Results}

There were 14 physicians that performed 137 procedures with EndoRings, and we identified 127 control patients. Thus, 10 procedures could not be matched for all criteria going back 2 years in our procedure database. For both EndoRings and the controls, $61 \%$ of subjects were female and $65 \%$ had screening as the indication, with the remainder being polyp surveillance

\section{$(\triangleright$ Table 1$)$.}

The PDR was $66 \%$ with EndoRings and $71 \%$ in controls ( $P=$ 0.34 ) ( $\triangleright$ Table 2 ). The ADR (fraction of patients with $\geq 1$ conventional adenoma) was $44 \%$ with EndoRings and $39 \%$ with controls $(P=0.39)$. At least one sessile serrated polyp (SSP) was detected in $11 \%$ of subjects with EndoRings and $9 \%$ of controls $(P=0.58)$. The polyp mean detection rate (standard deviation) expressed as PPC with EndoRings was 2.1 (2.9), versus 1.9

- Table 2 Polyp and adenoma detection rates and insertion and withdrawal times in subjects with EndoRings and controls.

\begin{tabular}{|l|c|c|}
\hline & EndoRings & Controls \\
\hline Number of subjects & 137 & 127 \\
\hline Polyp detection rate & $66 \%$ & $71 \%$ \\
\hline Polyps per colonoscopy (SD) & $2.1(2.9)$ & $1.9(2.8)$ \\
\hline Adenoma detection rate & $44 \%$ & $39 \%$ \\
\hline Adenomas per colonoscopy (SD) & $1.2(2.3)$ & $0.9(1.6)$ \\
\hline At least one sessile serrate polyp & $11 \%$ & $9 \%$ \\
\hline Mean insertion time (SD) in minutes & $6.2(3.2)$ & $6.6(6.7)$ \\
\hline Mean withdrawal time (SD) with no polyps in minutes & $9.2(3.1)$ & $9.9(3.9)$ \\
\hline Mean withdrawal time (SD) all patients in minutes & $12.5(5.3)$ & $16.1(10.3)$ \\
\hline SD, standard deviation & & 0.39 \\
\hline
\end{tabular}


(2.8) for controls $(P=0.60)$. The mean number of adenomas per colonoscopy (APC) with EndoRings was 1.2 (2.3) versus 0.9 (1.6); $P=0.055$. The mean number of SSPs per colonoscopy with EndoRings was $0.2(0.6)$ versus $0.1(0.6)$ in controls; $P=$ 0.61 .

Mean insertion time (standard deviation) with EndoRings was 6.2 (3.2) minutes versus 6.6 (6.7) minutes in the control group; $P=0.81$. Mean withdrawal time in subjects with no polyps was 9.2 (3.1) minutes with EndoRings versus 9.9 (3.9) minutes in controls; $P=0.65$. However, when all subjects were considered, mean withdrawal time was 12.2 (5.3) minutes with EndoRings versus 16.1 (10.3) minutes without; $P=0.0005$.

\section{Discussion}

The primary focus of use of mucosal exposure devices such as EndoRings, Endocuff, and a cap on the colonoscope tip is to improve detection of colorectal polyps. EndoRings has been shown in a randomized tandem study to improve polyp detection [13]. In this nonrandomized study we introduced EndoRings to members of our routine endoscopy staff to familiarize them with the device and test the effect of the device on colonoscopy performance with regard to detection, insertion time, and withdrawal time. Our study was not intended or powered for improvement in ADR, and no statistically significant differences in detection between groups was noted. For APC there was a trend in favor of EndoRings (2.1 versus 1.9; $P=0.055$ ). Certainly, no disadvantage of EndoRings was seen with regard to detection.

We also did not observe an adverse effect of EndoRings on speed of insertion for routine colonoscopy. Anecdotally, because EndoRings increases the diameter or profile of the colonoscope, it requires extra pushing to move it through some sigmoid colons that show narrowing, angulation, or muscular hypertrophy. Indeed, some patients with severe sigmoid diverticular disease must have the device removed to safely negotiate the sigmoid, in the same sense that these patients must sometimes have the adult colonoscope switched to a pediatric instrument, or even an ultra-thin instrument, to successfully negotiate the sigmoid colon [14]. We have also noted that EndoRings seems to stabilize the position of the colonoscope tip when loop reduction is occurring. This effect could prevent backward slippage of the colonoscope tip during loop reduction, and perhaps this or another effect counters the drag effect of the device in advancing through the sigmoid. Although the mechanism is not certain, we saw no downside of EndoRings for insertion efficiency.

Although not fully anticipated, we observed a reduction in total withdrawal time when EndoRings was used. We considered this observation sufficiently interesting, and potentially useful, particularly since there is a reasonable mechanism to explain the observation, that it warranted description for its hypothesis-generating value. In patients without polyps, this reduction in withdrawal time was from a mean of 9.85 minutes to 9.21 minutes, a difference that was not statistically significant and not very clinically significant. Although we did not instruct the endoscopist to expect any reduction in withdrawal time, we actually anticipated that a reduction in withdrawal time might occur in patients with no polyps. This was anticipated based on the senior author's previous experience with using devices such as EndoRings and Endocuff in other trials. Thus, these devices anecdotally appear to speed up the process of inspection during withdrawal. Much of the work of colonoscopy withdrawal is in probing the proximal sides of haustral folds with the colonoscope tip. Since devices such as EndoRings, Endocuff, and the short cap can be used to flatten these haustral folds, the endoscopist becomes satisfied that complete inspection has occurred in a shorter interval compared to performing colonoscopy without such a device. However, although the mean withdrawal time with EndoRings was numerically lower compared to examinations without the device in patients with no polyps, this difference was not significant. We might have failed to demonstrate a small but statistically significant reduction in withdrawal time with EndoRings when no polyps were present because there were few patients with no polyps. We suggest that additional study of this effect is warranted. When all subjects were considered, there was a very substantial reduction in withdrawal time from 16.05 minutes in the controls to 12.2 minutes in subjects with EndoRings. This result suggests that the main benefit in efficiency (if one exists) from use of a device such as EndoRings may be in stabilizing scope position at polyp locations during polypectomy. Anecdotally, endoscopists not uncommonly lose sight of a diminutive polyp between the time they first identify it and when they get the snare out the colonoscope tip and position it for resection. The rings of EndoRings seem to keep the colonoscope tip in stable position as this process occurs. Measuring the time for polypectomy would be useful in future studies of EndoRings and Endocuff.

A recent non-randomized study of clinical experience with Endocuff Vision (Arc Medical Design, Leeds, United Kingdom) supports these observations [15]. Among 410 colonoscopies divided into those performed before, during or after a pilot service evaluation, Endocuff was associated with a $16 \%$ increase in ADR. Remarkably, compared to the pre-cuff period there was shorter cecal intubation time with Endocuff ( 7 vs. $8 \mathrm{~min}, P=$ $0.002)$ and shorter withdrawal times when no polyps were evident ( 8 min $30 \mathrm{sec}$ vs. $12 \mathrm{~min}(P<0.01)$. Similar differences between cuff and no cuff were present when comparing the cuff period to the post cuff period. Thus, a non-randomized experience with Endocuff also suggests that distal attachments could produce substantial improvements in colonoscopy efficiency.

Our study has obvious limitations, in that it was not a randomized trial, and the controls were selected historically. Nevertheless, a comparison between the demographic features between the EndoRings subjects and the controls suggest that we effectively controlled for age, gender, and procedure indication. Further, there is at least a theoretical basis for how a device such as EndoRings could produce efficiencies during withdrawal. Finally, endoscopist behavior with the device may have been affected if they were early in their experience with EndoRings or because the devices were being used on a test basis and were free to the endoscopists. 


\section{Conclusion}

In summary, these data suggest that efficiencies in colonoscopy should be considered as primary endpoints for studies comparing EndoRings, Endocuff and cap colonoscopy to standard colonoscopy. We recommend that clinical trials with these devices target efficiencies in colonoscopy as a primary endpoint.

\section{Acknowledgements}

We acknowledge Ashlee J. Roell for assistance in preparing the manuscript.

Competing interests

Dr. Rex has received research support from EndoAid.

\section{References}

[1] Rex DK, Schoenfeld PS, Cohen J et al. Quality indicators for colonoscopy. Gastrointest Endosc 2015; 81: 31-53

[2] Corley D, Jensen CD, Marks AR et al. Adenoma detection rate and risk of colorectal cancer and death. N Engl J Med 2014; 370: 1298 - 1306

[3] Kaminski MF, Regula J, Kraszewska E et al. Quality indicators for colonoscopy and the risk of interval cancer. N Engl J Med 2010; 362: $1795-1803$

[4] Kaminski MF, Wieszczy P, Rupinski M et al. Increased rate of adenoma detection associates with reduced risk of colorectal cancer and death. Gastroenterology 2017; 153: 98 - 105
[5] Kahi C], Ballard D, Shah AS et al. Impact of a quarterly report card on colonoscopy quality measures. Gastrointest Endosc 2013; 77: 925 931

[6] Abdul-Baki H, Schoen RE, Dean K et al. Public reporting of colonoscopy quality is associated with an increase in endoscopist adenoma detection rate. Gastrointest Endosc 2015; 82: 676-682

[7] Radaelli F, Paggi S, Hassan C et al. Split-dose preparation for colonoscopy increases adenoma detection rate: a randomised controlled trial in an organised screening programme. Gut 2017; 66: $270-277$

[8] Subramanian V, Mannath J, Hawkey C] et al. High definition colonoscopy vs. standard video endoscopy for the detection of colonic polyps: a meta-analysis. Endoscopy 2011; 43: 499-505

[9] Pohl J, Schneider A, Vogell $\mathrm{H}$ et al. Pancolonic chromoendoscopy with indigo carmine versus standard colonoscopy for detection of neoplastic lesions: a randomised two-centre trial. Gut 2011; 60: 485 490

[10] Kahi C], Anderson JC, Waxman I et al. High-definition chromocolonoscopy vs. high-definition white light colonoscopy for average-risk colorectal cancer screening. Am J Gastroenterol 2010; 105: 1301 1307

[11] Hewett DG, Rex DK. Cap-fitted colonoscopy: a randomized, tandem colonoscopy study of adenoma miss rates. Gastrointest Endosc 2010; 72: $775-781$

[12] Chin M, Karnes W, Jamal MM et al. Use of the Endocuff during routine colonoscopy examination improves adenoma detection: A meta-analysis. World J Gastroenterol 2016; 22: 9642 - 9649

[13] Dik VK, Gralnek IM, Segol O et al. Multicenter, randomized, tandem evaluation of EndoRings colonoscopy-results of the CLEVER study. Endoscopy 2015; 47: $1151-1158$

[14] Bick BL VK, Rex DK. Regional center for complex colonoscopy: yield of neoplasia in patients with prior incomplete colonoscopy. Gastrointest Endosc 2016; 83: 1239 - 1244

[15] Tsiamoulos ZP, Misra R, Rameshshanker R et al. Impact of a new distal attachment on colonoscopy performance in an academic screening center. Gastrointest Endosc 2018; 87: 280-287 